Meeting Report

\title{
IBC Sixth Annual Meeting on Antibody Engineering Dec 4-6 1995, La Jolla, CA
}

\author{
Christine Metz, Ph.D. \\ The Picower Institute for Medical Research
}

During the early 1980s, monoclonal antibodies (mAbs) were envisioned by biomedical researchers and clinicians to be "magic bullets" in therapy. Although there are now many examples of in vitro $\mathrm{mAb}$ applications for diagnosis and monitoring of human diseases, only a handful of mAbs are being used as immunotherapeutic agents, and mostly in the treatment of cancer. Numerous obstacles including immunogenicity of murine antibodies, the impermeability of tissues to macromolecules, and the non-specific nature of some tumor antigens have hindered the success of traditional mAbs in the clinical setting. At this meeting, some of the most recent advances in antibody design and production were discussed.

\section{RECOMBINANT ANTIBODY TECHNOLOGY: OVERVIEW}

Recent advances in the fields of immunology and molecular biology have provided alternatives to the conventional, time-consuming murine mAb production technology which uses repeated animal immunization protocols and hybridoma generation. Antibody derivatives can now be rapidly expressed in the bacterium Escherichia coli via recombinant genetic techniques.

Phage display, a technique originally developed by McCafferty and co-workers (1), allows the expression of either the human or mouse variable (V) gene repertoire on the surface of bacteriophage as recombinant antibody-like molecules known as single chain variable fragments (scFvs). These scFvs consist of heavy $(\mathrm{H})$ and light $(\mathrm{L})$ chain $\mathrm{V}$ domains connected by a linker peptide (see Figure 1). Libraries of these Ig-like molecules are expressed on phage which can be selected in vitro by repeated panning procedures (using immobilized antigens) or by biotin selection (using antigens in solution). The
A.

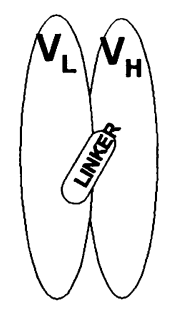

B.

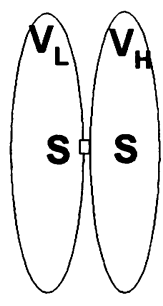

C.
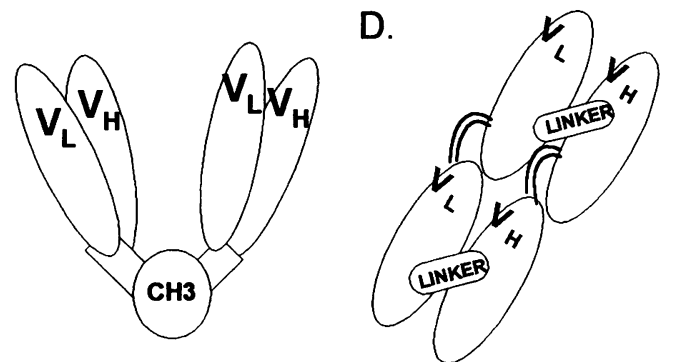

FIG. 1. Recombinant antibody-like molecules. (A) Single chain variable fragment (scFv); (B) Disulfide-bonded Fv (dsFvs); (C) Minibody; (D) Bifunctional Fv.

process of selection, screening and amplification is repeated 3-4 times to generate optimal scFvs for further development.

There are several advantages of recombinant antibody production via phage display over conventional murine mAb development: 1) scFvs can be generated within a few months, as compared to at least 1 year using traditional mouse mAb methods; 2 ) the antibodies do not need to be 'humanized' since human $\mathrm{V}$ gene regions are used to produce scFvs; 3 ) scFvs can be easily manipulated by genetic engineering methods to produce a more diverse panel of Ig-like molecules with improved affinities (Kd lnm-20nm); and 4) combinatorial libraries can be used to generate scFvs which recognize almost any epitope, including those on self antigens, toxic substances, and non-immunodominant epitopes. 
The major disadvantage of scFvs compared to traditional mAbs is their short half-life in vivo as they lack an Fc region. Several investigators are working on improving the stability of recombinant scFvs by altering the design of their structure. The construction and production of several types of modified scPvs were discussed, including disulfidebonded Fv fragments (dsFvs-Yoram Reiter, Ph.D., NCI, NIH), bifunctional antibodies (Peter Hudson, Ph.D., CSIRO, Australia), and miniantibodies (or minibodies-Andreas Phickthun, Ph.D., University of Zurich, Switzerland) (see Figure 1).

\section{THERAPEUTIC APPLICATIONS OF RECOMBINANT ANTIBODIES}

\section{Identification of Tumor Antigens for Vaccine Development}

One of the major pitfalls of conventional antibody development is the production of mAbs which recognize primarily immunodominant epitopes present on antigens. For example, conventional hybridoma technology produced primarily anti-ganglioside mAbs against melanoma cells as immunogen. Gangliosides are, however, ineffective for the production of a melanoma vaccine because they only induce $B$ cell immunity. Using phage display in combination with subtractive binding to normal melanocytes, Dorothee Herlyn, D.V.M. from the Wistar Institute identified several novel, melanoma-specific antigens. As these proteins induce both $\mathrm{T}$ and $\mathrm{B}$ cell immunity, they are potential candidates for the development of melanoma vaccines.

\section{Immunodiagnostics}

Gary Barsomian, Ph.D. and Diane Copeland, Ph.D. of Genzyme Corporation discussed the use of phage display for the generation of high affinity scFvs which recognize novel fetal cell-specific antigens. The commercially available anti-fetal cell mAb (anti-CD71) is not useful in genetic screening procedures because it does not effectively discriminate between fetal and circulating maternal blood cells. Using phage display, followed by a fetal liver cell enrichment step, high affinity scFvs specific only for fetal cells were generated. When compared to the commercially available mAb, these scFvs are ten fold more specific for fetal cells than maternal blood cells.

\section{Tumor Imaging}

One of the major disadvantages of recombinant scFvs is their short half life in vivo. However, Ann $\mathrm{Wu}, \mathrm{Ph} . \mathrm{D}$. and co-workers from the Beckman Research Institute of the City of Hope, have ex- ploited this 'disadvantage' to develop a panel of recombinant Ig-like molecules specific for the carcinoembryonic antigen (CEA) to be used in colon carcinoma imaging. Using nude mice bearing human colon carcinoma xenografts, the antiCEA ${ }^{123}$ I-labeled minibody exhibited high, specific tumor uptake, rapid blood clearance, and excellent camera imaging within four hours of administration. Use of the ${ }^{123}$ I-labeled anti-CEA minibody for tumor imaging of colon cancer patients has just been initiated.

\section{PRODUCTION OF "HUMANIZED" ANTIBODIES}

For repeated clinical applications, murine mAbs must be 'humanized' following their production by traditional hybridoma methods. Using genetic engineering techniques, the rodent variable or hypervariable gene regions are combined with the human constant gene regions. Although this process produces mAbs which can be administered to humans without severe immunogenic reactions, it is quite labor-intensive and timeconsuming. Aya Jakobovits, Ph.D. from Cell Genesys, Inc. discussed the production of human antibodies by mice genetically engineered with YACS (yeast activated chromosomes) containing human Igs. These mice lack mouse antibody genes and express human heavy and kappa light chain regions. Thus far, 'human' antibodies to almost every antigen tested have been produced by these mice. Similarly, Nils Lonberg, Ph.D. and co-workers from Genpharm International, have engineered a line of transgenic mice from Ig knockout mice which express fully human, high affinity IgGs.

\section{CONCLUSIONS}

The expression of both 'humanized' antibodies using genetically engineered mice and recombinant antibody-like molecules or scFvs using phage display represent two powerful techniques for the design and production of antibodies to a greater variety of antigens. Given these two powerful tools, researchers continue to strive for the production of antibodies that can be used in the clinical setting, for diagnostics, tumor imaging, and immunotherapy. Hopefully, this generation of antibodies will provide better aimed bullets.

\section{REFERENCES}

1. McCafferty J, Griffiths AD, Winter G, Chiswell DJ. (1990) Phage antibodies: filamentous phage displaying antibody variable domains. Nature 348: 552-554. 